This item was submitted to Loughborough's Research Repository by the author.

Items in Figshare are protected by copyright, with all rights reserved, unless otherwise indicated.

\title{
Utilising human performance criteria and computer simulation to design a martial arts kicking robot with increased biofidelity
}

\section{PLEASE CITE THE PUBLISHED VERSION}

http://dx.doi.org/10.1177/1754337112439275

PUBLISHER

(c) Sage

VERSION

AM (Accepted Manuscript)

LICENCE

CC BY-NC-ND 4.0

\section{REPOSITORY RECORD}

Tsui, Felix, and Matthew T.G. Pain. 2019. "Utilising Human Performance Criteria and Computer Simulation to Design a Martial Arts Kicking Robot with Increased Biofidelity”. figshare. https://hdl.handle.net/2134/12540. 
This item was submitted to Loughborough's Institutional Repository (https://dspace.lboro.ac.uk/) by the author and is made available under the following Creative Commons Licence conditions.

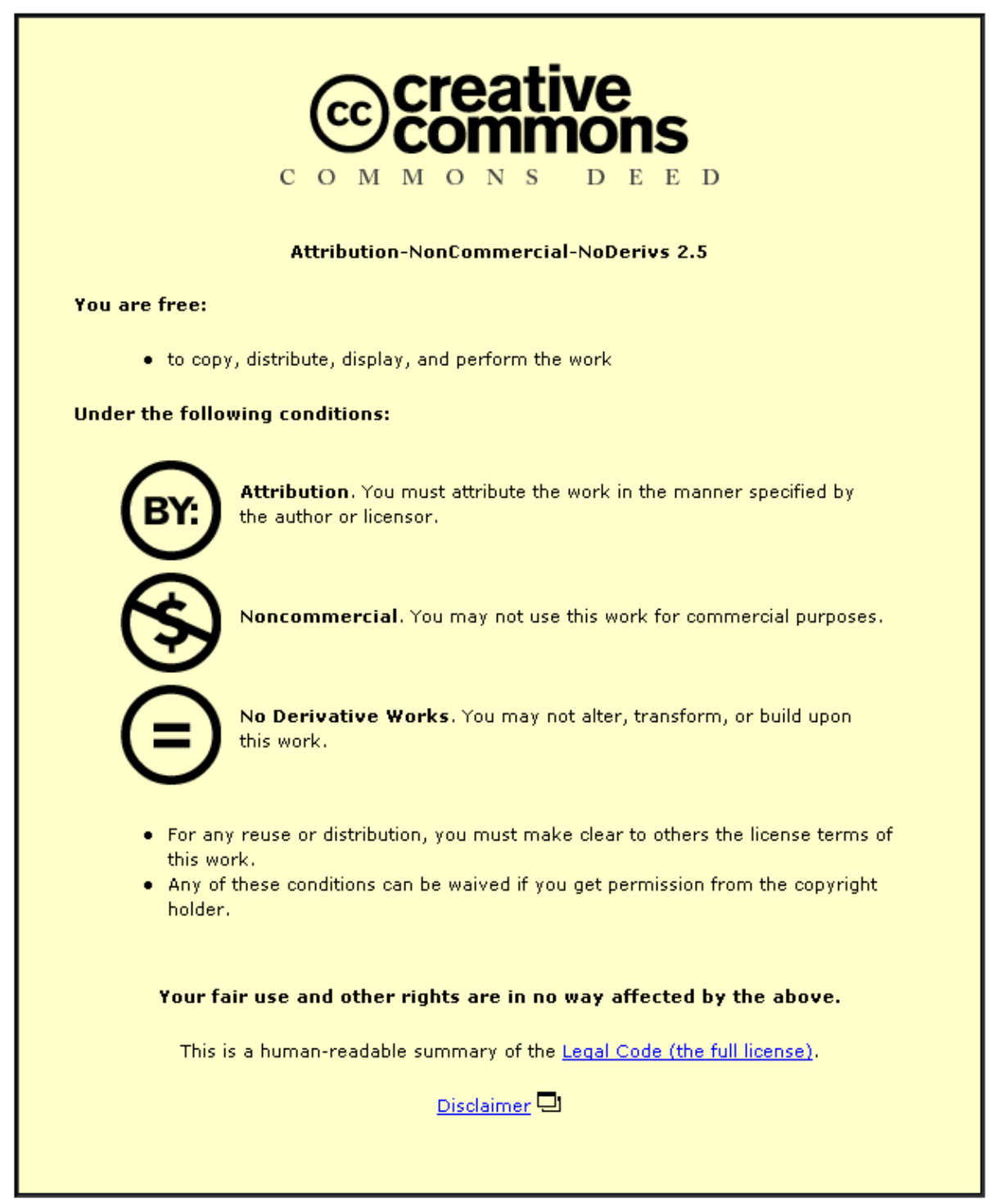

For the full text of this licence, please go to: http://creativecommons.org/licenses/by-nc-nd/2.5/ 
Proceedings of the Institution of Mechanical Engineers, Part P, Journal of Sports Engineering and Technology, 226, 244-252.

\title{
Utilising human performance criteria and computer simulation to design a martial arts kicking robot with increased biofidelity
}

\author{
${ }^{1}$ Felix Tsui, and ${ }^{2}$ Matthew TG Pain \\ ${ }^{1}$ Wolfson School of Mechanical and Manufacturing Engineering, Loughborough University, \\ Loughborough, LE11 3TU, UK \\ ${ }^{2}$ School of Sport, Exercise and Health Sciences, Loughborough University, Loughborough, \\ LE11 3TU, UK
}

\begin{abstract}
The rules and regulations of Taekwondo stipulate how the sport must be played and the necessary personal protective equipment (PPE). As such, PPE performance under controlled rigid drop-tests is also outlined. Unfortunately, these impacts do not replicate human loading effectively making conclusions about their performance unknown. However, it may be possible to use human kinematic data to improve the biofidelity of current impactors, including a current single-segment martial arts kicking robot (STIMAK). Five martial artists performed a series of roundhouse kicks whilst reflective markers on the kicking leg and pelvis were used to track hip, knee, ankle and foot positions. Using specific STIMAK robot parameters, computer simulation was used to model STIMAK performance (1-SM) and to form a multi-segment, multi-joint model to match human kinematic data (3-SM). The 3-SM was found to produce similar kinematics to human performance whilst reducing the overall effective mass at impact, motor torque and stress concentration magnitudes in the leg when compared to the 1SM. This study suggested that human performances could be used to improve current mechanical testing techniques without introducing much complexity to improve the external validity of protective equipment evaluation testing.
\end{abstract}

Keywords: biofidelity, hogu, mechanical impactor, PPE 


\section{Introduction}

In professional sport, governing bodies stipulate the rules and regulations for match play thus defining the allowable playing actions within the sport and controlling the conditions for which the game will be played. As such, there are standards for how the playing surface and equipment should perform, and how each should interact with the player to improve performance and minimise injury. Whilst there has been substantial research into improving the playing conditions to increase performance, research into personal protective equipment (PPE) is lacking. One of the limiting factors in advancing this research is the low biofidelity of the impactors used to evaluate PPE performance.

Rather than having actual athletes generate loads to test the suitability of a sports surface or PPE, mechanical devices are used as surrogates to develop impacts. In sports surfaces, a Clegg hammer or a Berlin Artificial-Athlete (BAA) are used to mimic foot-strike during running. Each of these apparatus' releases a rigid mass from a fixed height and examines the impact absorption of any surface with respect to a stiff surface such as concrete. Whilst the Clegg is purely a drop-mass test only, the BAA has increased biofidelity as it introduces a spring at impact to increase impact compliance and contact duration. Despite the high repeatability of both tests, the correlations between mechanical tests and the impact forces which occur during running are low [1]. However, recent research has utilised industrial robots with foot prostheses to generate repeatable ground contact forces that emulate human data [2]. Similarly, surrogate mechanical models of soccer kicks such as a rigid mass dropped from a specific height [3-6] or a mass on a pendulum [7] have shown low biofidelity in the testing of soccer shin guards. British Standards testing of PPE in martial arts [8] also specify the use of a drop test for their evaluation protocol. Whilst these tests are consistent and repeatable, the impactors artificially matched either the impact force or impact energy with an incorrect loading rate and/or effective mass. Since kicks during match conditions feature two non-linear, visco-elastic bodies instead of two rigid bodies, these rigid impactor tests only provided information on the dynamic material properties of the pad under a specific loading regime and not the protection afforded to the user [9].

Research into the effectiveness of rugby shoulder pads has also highlighted the importance of the impactor when evaluating PPE performance. Rigid impactors deformed the foam materials in the shoulder pad in a point-elastic manner providing minimal dispersion of the load [10]. Thus, the force reduction observed was a function of the increased contact time during impact as the pad deformed resulting in lower deceleration values. In contrast, Milburn, Wilson \& Chalmers [11] used a medicine ball, considered to be much more representative of soft tissue, to impact various shoulder pads and did not find a significant improvement in force reduction. In these tests, impact energy was not only dissipated within the pad, but also in deforming the impactor and it was likely that this accounted for its impact force response. As such, the benefit of using shoulder pads as protectors against soft tissue trauma has been heavily debated [10]. It is clear that impactors should match impact scenarios from match conditions as closely as possible if there is to be increased validity in PPE evaluation procedures. 
The Sports Technology Institute (STI) single-segment martial arts kicking (STIMAK) robot was constructed at Loughborough University to more closely replicate the velocities and effective mass of roundhouse kicks [12]. The roundhouse kick has been found to be the most frequently used (over 50\%) attacking technique in Taekwondo accounting for roughly $89 \%$ of all points scored [13]. The design of the STIMAK robot was similar to the STI soccer kicking robot [14] and consisted of two tapered beams made of high-strength aerospace grade aluminium alloy 7075-T6 connected by vertical struts. Leg mass, $12.4 \mathrm{~kg}$, leg length, $0.95 \mathrm{~m}$, and moment of inertia (Mol), $3.04 \mathrm{~kg} \cdot \mathrm{m}^{2}$, were based on anthropometric data of a $50^{\text {th }}$ percentile male $[15,16]$. A 9.2-kW Lenze (Bedford, UK) geared servo-motor housed in a steel frame was used to drive the leg and was capable of generating an end effector velocity of 25 $\mathrm{ms}^{-1}$ within $270^{\circ}$ leg rotation to avoid contact with its housing frame. This velocity is greater than the highest peak velocity $\left(18.83 \pm 5.81 \mathrm{~ms}^{-1}\right)$ measured for roundhouse kicks in previous studies [17]. The motor also featured a torque limiter to protect the motor and a clutch to ensure that the hemispherical nylon end effector $(d=0.15 \mathrm{~m})$ was not driven through impact.

Despite improving upon previous impactors, there were several limitations to its design. Firstly, during roundhouse kicks, toe and ankle velocities have been found to be significantly greater than the knee and hip at impact implying that the shank and foot were mainly responsible for the momentum transferred at impact [18]. The STIMAK robot did not account for this co-ordination in limb segments resulting in higher impact forces than a multi-segment limb due to a greater effective mass being involved during impact. In human movement, the co-ordination of limb segments is one of the primary means of controlling the intensity of a given impact, not the use of external deformable materials (i.e. padding). For example, runners adjust their leg kinematics to different surface stiffness' to control their ground reaction forces [19]. A secondary means of controlling impact intensity is through wobbling masses or soft tissue movement. However, the STIMAK robot was composed entirely of rigid mass which was its second limitation. Whilst it is difficult to model soft tissue response, particularly since it depends on its stiffness (i.e. activation and tension of the muscles), it has been found to reduce impact force, dissipate impact energy and reduce joint loads [20-22]. In particular, the energy dissipative properties of the most distal segment have been found to have the largest effect, when normalised to mass, on impact intensity [21]. As such, separating the STIMAK robot into multiple-segments could lessen the effect of the poor force attenuation properties of the current materials and reduce the limitations to the more distal segments (i.e. foot and shank) only. Lastly, the impact properties of the end effector and rigid joint attaching the end effector to the leg did not reflect the compliance found in the human foot or ankle joints. Joint torques are variable throughout a given movement so the resistance to rotation should be expected to change and not remain constant as found in the STIMAK robot.

Whilst drop tests and rotational impactors such as a pendulum or the singlesegment robot have low biofidelity, increasing the number of segments may not be realistic. Schempf [23] used a combination of active and spring-assisted actuator modules at multiple joints to control a three-segment kicking robot. Activation and coordination of each component was based on human performance in order to replicate realistic joint angles and angular velocities. In spite of its ability to mimic human motion, 
the significant cost and complexity made its operation brief as it suffered from overheating and mechanical failures in the gearing. As such, a balance between the artificial drop, or pendulum, tests and the exact simulation of human movement is necessary. Mathematical models $[18,24]$ have shown that multi-segment mechanical kick models can be simple yet provide insight into kicking and PPE performance.

The aim of this paper was to demonstrate that utilising human kinematic data and some of the principles of human impact mechanics described above can be used to improve upon a current martial arts kicking robot. A simple multi-segment computer simulation model is used to match the key parameters of human performance. This has implications for future methodologies for designing surrogate mechanical impactors in the evaluation of sports PPE and beyond.

\section{Methods}

\section{Kinematic Kicking Data}

Five physically active male martial artists $\left(1^{\text {st }}\right.$ dan or higher, $27.6 \pm 8.8 \mathrm{yrs}, 77.8 \pm$ $10.8 \mathrm{~kg}, 1.77 \pm 0.06 \mathrm{~m}$ ) provided informed voluntary consent to participate in accordance with the protocol approved by the Loughborough University Ethical Advisory Committee. After a self-selected warm-up, each subject performed twenty front-leg roundhouse kicks with their dominant leg. In each kick, subjects were asked to generate maximal impact force when striking the chest of a commercially available martial arts dummy, BOBXL (Century LLC, Oklahoma, USA). Kinematic data were obtained using nine Vicon MX cameras at $250 \mathrm{~Hz}$ to track the position of eleven markers on the kicking leg and pelvis. Markers were used to identify joint centres and axes of rotation for the hip, knee and ankle. Toe markers were also used to determine impact velocity perpendicular to the chest of BOBXL.

In each successful kick, one in which all of the markers could be reconstructed reliably, the frame of first contact was identified and used to temporally align the performances of each kick. Average hip, knee and ankle angles were calculated in the axis of flexion-extension for each frame. Standard deviations at each frame were used to set upper and lower bounds to account for the variations in human performance. Joint angles were compared to literature to ensure that they were representative of elite performances.

\section{Modelling}

Computer simulation modelling was performed using Visual Nastran 4D (MSC.Software $\mathrm{GmbH}$, Munich, Germany) in two parts. Part one modelled the STIMAK robot using physical constraints from its design, which included the motor, housing frame, inertia parameters of the single segment and properties of the end effector, to produce a single-segment computer-simulated kicking model (1-SM). Part two replaced the single-segment leg and end effector with multiple segments, including revolute joints and spring-damper mechanisms at each joint, to produce a three-segment computersimulated kicking model (3-SM). Parameters of the motor and spring dampers, along with the times at which each were active, were varied to generate a kicking action that matched the kinematics of human performance. Results from the 3-SM were compared to measured human joint angle data and human-on-human impact force data. Impact 
forces and time to peak force (TTPF) were also compared to the results of the 1-SM to determine its biofidelity relative to the STIMAK robot and human performance. For each computer model, three velocities $\left(12.0,14.0\right.$ and $\left.16.0 \mathrm{~ms}^{-1}\right)$ were simulated whilst using a Hertzian contact model with $1 \mathrm{e}+04$ iterations per second and positional tolerances of $1 \mathrm{e}-04 \mathrm{~m}$.

The 1-SM was designed to have a single segment with the same length, mass and moment of inertia as the STIMAK robot. End effector velocity was controlled using motor torque and its activation time defined as the duration at which the motor was active relative to the initiation of movement at time zero. Torque magnitudes were minimised to produce the least strain on the motor by maximising activation time and ensuring the use of the entire range for the rotation angle of the leg (i.e. $270^{\circ}$ ). When the motor was inactive (i.e. with the clutch disengaged), the leg was left to swing at its current angular velocity about a revolute joint.

The 3-SM consisted of a motor (hip) and two time-delayed angular springdampers at the knee and ankle. Movement of the 3-SM was generated by the motor which, similar to the 1-SM, remained active until just before contact. The knee angle was first fixed in full extension $\left(180^{\circ}\right)$ until allowed to freely rotate, about a revolute joint, where the conserved angular momentum would cause the knee to 'flex' as the hip was 'flexing' (i.e. motor was active). As the knee approached maximum flexion, a timedelayed spring-damper was engaged to simulate the knee extensors. The ankle remained fixed until just prior to impact where its time-delayed spring-damper would engage. Motor torque and time-delays for both spring-dampers at the knee and ankle were based on iteratively matching the co-ordination of joint angles in the flexionextension axes obtained from the kinematic data.

The computer-simulated anvil was a modified, more basic version of the Lobdell chest model [25] consisting of only one linear spring-damper connecting two masses ( $\mathrm{m}$ $=5$ and $50 \mathrm{~kg}$ ). On impact, the amount of thoracic deformation, movement of the lighter mass relative to the larger mass, was a function of the spring-damper parameters and was constrained to translation only along the axis of impact. Spring stiffness $(k=200$ $\left.\mathrm{N} \cdot \mathrm{m}^{-1}\right)$ and damping $\left(\mathrm{c}=100 \mathrm{~N} \cdot \mathrm{s} \cdot \mathrm{m}^{-1}\right)$ were set to allow for an acceptable amount of deformation whilst absorbing a fraction of the impact energy and not returning it to the foot. These parameters were not optimised to replicate the Lobdell model or BOBXL but were set to produce realistic impact conditions such as impact force in the 3-SM model. 


\section{Results}

\section{Kinematic Kick Data}

An average of all successful kick performances is reported in Table 1 with standard deviations as well as minimum and maximum values. A summary of previous research reporting the same variables for other elite martial artists are shown in Table 2.

Table 1. Summarised kick results from all subjects.

\begin{tabular}{l|cccc}
\hline \multicolumn{1}{c|}{ Parameter } & \multirow{2}{*}{ Average } & \multirow{2}{*}{ SD } & \multicolumn{2}{c}{ Range } \\
& & & min & max \\
\hline Impact velocity $\left(\mathbf{m s}^{-1}\right)$ & 12.8 & 1.2 & 10.8 & 14.9 \\
Maximum hip angular velocity $\left({ }^{\circ} \cdot \mathbf{s}^{-1}\right)$ & 530 & 150 & 272 & 806 \\
Maximum knee angular velocity $\left(^{\circ} \cdot \mathbf{s}^{-1}\right)$ & 1448 & 109 & 1204 & 1791 \\
Overall change in knee angle $\left({ }^{\circ}\right)$ & 69.0 & 6.5 & 51.8 & 80.3 \\
\hline
\end{tabular}

Table 2. Impact velocities and joint angular velocities of a roundhouse kick from literature.

\begin{tabular}{lccc}
\hline \multirow{1}{*}{ Parameter } & \multicolumn{3}{c}{ Study } \\
\cline { 2 - 4 } & $\begin{array}{c}\text { Kong et al. } \\
{[17]}\end{array}$ & $\begin{array}{c}\text { Tang et al. } \\
{[26]}\end{array}$ & $\begin{array}{c}\text { O'Sullivan et al. } \\
{[27]}\end{array}$ \\
\hline $\begin{array}{l}\text { Impact Velocity }\left(\mathbf{m s}^{-1}\right) \\
\begin{array}{l}\text { Maximum hip angular } \\
\text { velocity }\left({ }^{\circ} \cdot \mathbf{s}-1\right)\end{array}\end{array}$ & $18.8 \pm 5.8$ & $17.6 \pm 1.2$ & $17.7 \pm 1.7$ \\
$\begin{array}{l}\text { Maximum knee angular } \\
\text { velocity }\left({ }^{\circ} \cdot \mathbf{s}-1\right)\end{array}$ & $913 \pm 308$ & $1737 \pm 322$ & $1586 \pm 181$ \\
\hline
\end{tabular}

Overall, impact velocities were lower than the average range reported [17, 26, 27]. However, these authors had reported maximum values at any time during the kick and not those at impact. Kong et al. [17] and Kim et al. [28] had shown that impact velocities could be considerably less (as much as $10 \mathrm{~ms}^{-1}$ ) than the maximum measured during the kick, a trend also observed in the kicks performed in this study. Angular velocities at the hip and knee were found to compare well with other two studies [26, 27]. Similarly, the overall change in knee angle from maximum knee flexion to maximum extension compared well to the values of $67.6 \pm 22.9^{\circ}$ [17] and $63.4 \pm 8.5$ [27] previously reported.

\section{Matching Kinematics}

In the 1-SM, motor torque values increased to produce increasing impact velocities (Table 3). In addition, activation times decreased as the time it took the leg to travel the same angular distance (i.e. $270^{\circ}$ ) was decreased at higher torques.

Table 3 Model parameters for the1-SM at three different velocities.

\begin{tabular}{cccc}
\hline $\begin{array}{c}\text { Velocity } \\
\left(\mathbf{m} \cdot \mathbf{s}^{-1}\right)\end{array}$ & $\begin{array}{c}\text { Angular Velocity } \\
\left({ }^{(} \cdot \mathbf{s}^{-1}\right)\end{array}$ & $\begin{array}{c}\text { Torque } \\
(\mathbf{N} \cdot \mathbf{m})\end{array}$ & $\begin{array}{c}\text { Activation Time } \\
(\mathbf{m s})\end{array}$ \\
\hline 12.0 & 837 & 63 & $<0.39$ \\
14.0 & 983 & 97 & $<0.354$ \\
16.0 & 1090 & 135 & $<0.321$ \\
\hline
\end{tabular}


A comparison of joint angles for the hip, knee and ankle for an average human performance and a $16 \mathrm{~ms}^{-1}$ kick performed by the 3-SM are shown in Figure 1. The frames of impact were normalised and set to $t=0 \mathrm{~s}$ for human and simulated performances (impact at $t=0.385 \mathrm{~s}$ for the model parameters). At the hip, the rate of hip extension pre-impact and hip flexion post-impact in the 3-SM were greater than the human performances. At the knee, joint angles pre-impact were similar though there was slightly greater knee flexion found in human performance. Post-impact, the human knee continued extending for a longer duration resulting in greater overall knee extension. This phenomenon was more pronounced (i.e. larger post-impact extension) in the 3-SM. At the ankle, only the conditions at impact were similar as both performances exhibited rapid plantar-flexion before returning to its pre-contact angle.
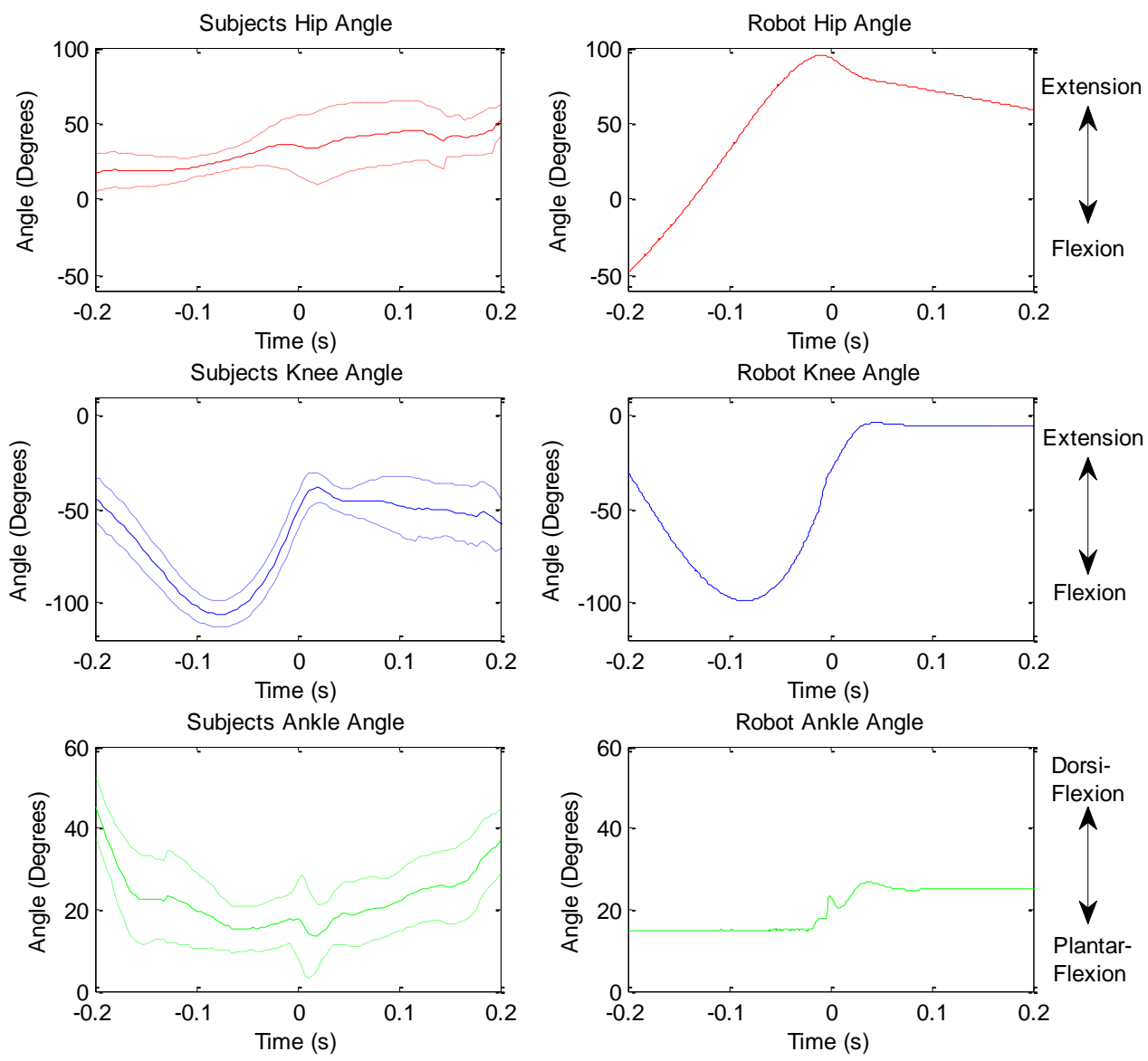

Figure 1. Comparison of hip, knee, and ankle angles between human performance and 3-SM for $200 \mathrm{~ms}$ before and after contact (Time 0). As reference, the knee and ankle joints were considered in its anatomical position at $0^{\circ}$ and $90^{\circ}$, respectively.

Model parameters of the 3-SM required to achieve the performance described are shown in Table 4. Motor torques were lower in the 3-SM models than the 1-SM as the 3-SM relied on the co-ordination of the limb segments to produce its end effector 
velocity. In the $1-\mathrm{SM}$, the linear velocity of the knee position was $\sim 45 \%$ of the impactor velocity at impact whilst this was reduced to just $\sim 8 \%$ in the $3-S M$. At all simulated impact velocities, the 3-SM produced lower peak forces compared to the 1-SM though this was coupled with decreased time to peak force (TTPF) (Table 5).

Table 4. Model parameters for the 3-SM kicks at three different velocities. Temporal parameters are reported relative to the initiation of movement (from rest at time zero).

\begin{tabular}{|c|c|c|c|c|c|}
\hline \multirow{2}{*}{ Joint } & \multirow{2}{*}{\multicolumn{2}{|c|}{ Parameters }} & \multicolumn{3}{|c|}{ Velocity } \\
\hline & & & 12.0 & 14.0 & 16.0 \\
\hline \multirow{6}{*}{ Hip } & \multicolumn{2}{|c|}{ Initial angle $\left({ }^{\circ}\right)$} & -175 & -180 & -180 \\
\hline & \multicolumn{2}{|c|}{ Maximum hip angular velocity $\left({ }^{\circ} \cdot \mathbf{s}^{-1}\right)$} & 782 & 870 & 936 \\
\hline & \multicolumn{2}{|c|}{ Hip angular velocity at impact $\left({ }^{\circ} \cdot s^{-1}\right)$} & 64.9 & 41.9 & 179.0 \\
\hline & \multirow{2}{*}{ Torque } & $\begin{array}{r}\text { Value }(\mathrm{N} \cdot \mathrm{m}) \\
\end{array}$ & 56 & 70 & 82 \\
\hline & & Active (s) & $<0.49$ & $<0.42$ & $<0.38$ \\
\hline & Revolute Joint & Active (s) & $>0.48$ & $>0.41$ & $>0.37$ \\
\hline \multirow{10}{*}{ Knee } & \multirow{2}{*}{\multicolumn{2}{|c|}{$\begin{aligned} \text { Initial angle }\left(^{\circ}\right) \\
\text { Maximum change in knee angle }\left(^{\circ}\right)\end{aligned}$}} & 0 & 0 & 0 \\
\hline & & & 58.8 & 62.6 & 69.7 \\
\hline & \multicolumn{2}{|c|}{ Time between knee flexion \& impact (s) } & 0.086 & 0.079 & 0.079 \\
\hline & \multicolumn{2}{|c|}{ Maximum knee angular velocity $\left({ }^{\circ} \cdot \mathbf{s}^{-1}\right)$} & 1730 & 1870 & 2350 \\
\hline & Fixed Joint & Active (s) & $<0.12$ & $<0.12$ & $<0.115$ \\
\hline & Revolute Joint & Active (s) & always & always & always \\
\hline & \multirow{4}{*}{ Spring } & Resting Length $\left(^{\circ}\right)$ & -5 & -5 & -5 \\
\hline & & Spring Constant $\left(\mathrm{N} \cdot \mathrm{m} \cdot \cdot^{\circ-1}\right)$ & 11.5 & 19 & 22 \\
\hline & & Damper (N.s. $\left.\mathbf{m}^{-1}\right)$ & 0.31 & 0.31 & 0.31 \\
\hline & & Active $\left({ }^{\circ} \cdot \mathrm{s}^{-1}\right)$ & $>0.431$ & $>0.4$ & $>0.367$ \\
\hline \multirow{6}{*}{ Ankle } & & Initial angle $\left({ }^{\circ} \cdot \mathrm{s}^{-1}\right)$ & 15 & 15 & 15 \\
\hline & Fixed Joint & Active (s) & $<0.402$ & $<0.395$ & $<0.361$ \\
\hline & \multirow{4}{*}{ Spring } & Resting Length $\left({ }^{\circ}\right)$ & 10 & 10 & 10 \\
\hline & & Spring Constant $\left(\mathrm{N} \cdot \mathrm{m}^{\mathrm{o}^{-1}}\right)$ & 10 & 10 & 10 \\
\hline & & Damper (N.s. $\left.\mathrm{m}^{-1}\right)$ & 1 & 1 & 1 \\
\hline & & Active $\left({ }^{\circ} \cdot \mathrm{s}^{-1}\right)$ & $>0.401$ & $>0.395$ & $>0.360$ \\
\hline
\end{tabular}

Table 5.Comparison of impact force and time to peak force (TTPF) for the 1-SM and 3$\mathrm{SM}$ for a roundhouse kick.

\begin{tabular}{ccccc}
\hline \multirow{2}{*}{ Velocity $\left(\mathbf{m s}^{-1}\right)$} & \multicolumn{2}{c}{ Simulated 1-SM } & \multicolumn{2}{c}{ Simulated 3-SM } \\
& Impact Force (N) & TTPF (ms) & Impact Force (N) & TTPF (ms) \\
\hline 12 & 5620 & 3 & 4400 & 1 \\
14 & 6380 & 3 & 4540 & 1 \\
16 & 6810 & 3 & 4850 & 1 \\
\hline
\end{tabular}

\section{Discussion}

The aim of this study was to show that human biomechanical data could be used to improve upon existing mechanical impactors. In particular, an existing martial arts robot was evaluated and modifications were proposed to increase its biofidelity through the use of computer simulation. To determine its effectiveness in achieving this aim, 
two main areas were analysed. First, the biofidelity of the 3-SM robot is discussed relative to the STIMAK and 1-SM robots. Second, the performance of the 3-SM is compared to joint kinematics of human performance.

\section{3-SM biofidelity relative to STIMAK robot and 1-SM}

As shown in Table 5, the 3-SM produced much lower impact forces at each impact velocity compared to the 1-SM. In a $16 \mathrm{~ms}^{-1} \mathrm{kick}$, the $1-\mathrm{SM}$ had a $12.4-\mathrm{kg}$ rigid leg moving at an angular velocity of $1090^{\circ} \cdot \mathrm{s}^{-1}$ at impact. As such, its entire rotational momentum was converted into linear momentum at initiation of impact contributing to an increase in impact force when compared to the 3-SM which essentially had a lighter mass (4.7- $\mathrm{kg}$ shank plus foot) travelling at a much higher angular velocity $\left(2350^{\circ} \cdot \mathrm{s}^{-1}\right)$. Moreover, by not rigidly attaching segments at each joint, distal segments were free to rotate relative to their more proximal segments creating compliance in the system. A concomitant decrease in effective mass (through co-ordinated limb segments) and an increase in compliance increases the overall biofidelity of 3-SM. The importance of this compliance was also evident in the rapid plantar-flexion at impact but this will be discussed in the next section.

Another advantage of the 3-SM when compared to the 1-SM was that it generated lower magnitudes of stress concentration during impact (Figure 2). A simple finite-element analysis of both computer simulation models revealed significantly greater stress levels during impact for the 1-SM (2.6 MPa) versus the 3-SM (0.82 MPa). As such, the mass of the 1-SM could not be reduced to decrease the effective mass as it would risk damage to the STIMAK leg segment it was replicating.

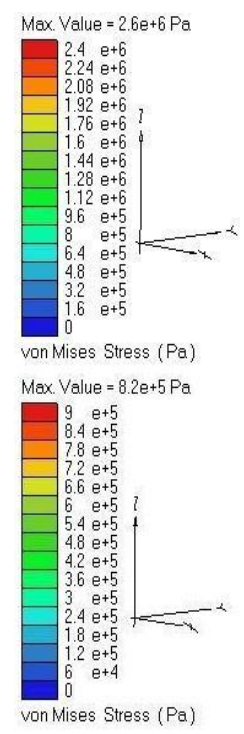

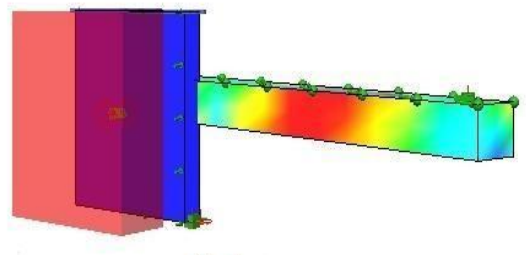

(a)

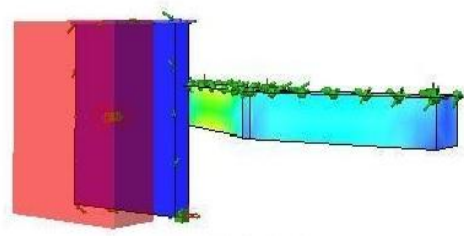

(c)

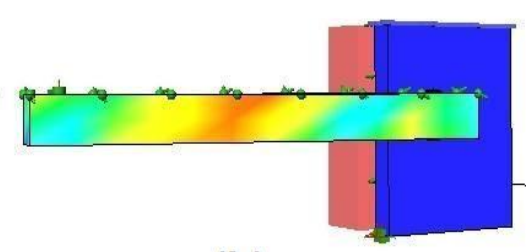

(b)

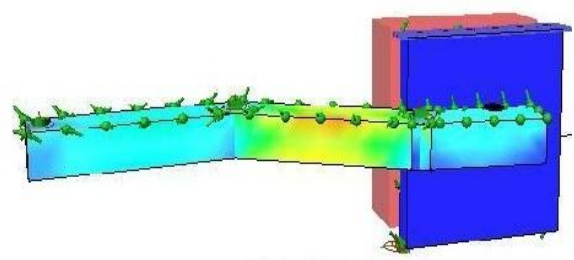

(d)

Figure 2. Finite element analysis at $16 \mathrm{~ms}^{-1}$ for the 1-SM: a) posterior view, b) anterior view; and the 3-SM: a) posterior view, b) anterior view

At first analysis, it might be expected that during a less violent impact generated with the 3-SM, the TTPF would not be much lower when compared to the 1-SM as a more compliant system would be expected to have a greater TTPF than a stiff, rigid 
system. However, as the leg was free-swinging at impact and the foot was free to rotate about the ankle, its peak deceleration would occur more quickly. This led to higher impact loading rates in the 3-SM, but its overall impact impulse was less than the 1-SM.

\section{3-SM biofidelity relative to Human Performance}

The 3-SM was able to exhibit the same kinetic chain or co-ordination of limb segments evident in human performance. Initially, movement of the 3-SM leg was generated with accelerations at the hip with the knee and ankle lagging behind. After approaching $\sim 100^{\circ}$ flexion, knee extension occurred producing a whip-like movement of the shank and foot as the thigh began to decelerate. This extension continued through impact and was coupled with slight dorsi-flexion pre-impact to prepare the foot for impact and rapid plantar-flexion at impact.

Whilst the co-ordination of the 3-SM limb segments matched the human performance visually, hip and ankle angles suggested a model of low-biofidelity. The main issue was that the 3-SM was a planar model (flexion-extension) of a multi-planar human movement. This was especially evident at the hip which in human movement normally uses hip adduction and internal rotation to generate acceleration of the distal limb segments and has been measured at $26.3 \pm 9.1^{\circ}$ and $37.1 \pm 7.9^{\circ}$, respectively [28]. Moreover, humans use changes in hip angular joint accelerations to help achieve maximum end effector velocity whilst the 3-SM was constrained to using constant torque output. As such, it was physically impossible to match the magnitude and rate of change in joint accelerations at the hip which help to explain the differences in hip flexion-extension angles shown in Figure 2. Moreover, the 3-SM hip angle was measured relative to an arbitrary axis instead of relative to the torso as it was in kinematic data. The other joints did not suffer from this limitation as they were measured relative to the more proximal segment. The co-ordination of the ankle joint was also dissimilar as it was fixed during the kick until just prior to impact. The contribution of the ankle was mainly to introduce compliance into the system by allowing the end effector to rotate about its more proximal segments (i.e. plantar-flexion) during impact. Human performance did not exhibit any large changes in ankle angle preimpact so this was thought to be an acceptable simplification. Whilst a multi-planar model may be ideal, significant cost and difficulties in achieving the desired accuracy may not make the risk worthwhile [23].

Despite the differences in the kinematic performance at the hip and ankle, there was evidence to suggest that the 3-SM was an acceptable simplification. Serina \& Lieu [18] reported linear velocities at the hip and knee to be $3 \%$ and $7 \%$ of the toe velocity at impact, respectively, whilst Tang et al. [26] found maximum hip and knee linear velocities during the swing phase of the kick to be $18 \%$ and $47 \%$ of toe impact velocity. In the 3-SM, the hip was fixed so linear hip velocity at all times was $0 \mathrm{~ms}^{-1}$. Meanwhile, hip angular velocity at impact was $3.1 \mathrm{rad} \cdot \mathrm{s}^{-1}$ equating to a $1.3 \mathrm{~ms}^{-1}$ linear knee velocity or $8.4 \%$ of the toe linear velocity in a $16 \mathrm{~ms}^{-1}$ kick. In addition, the maximum hip angular velocity was $16 \mathrm{rad} \cdot \mathrm{s}^{-1}$ producing a maximum swing phase velocity at the knee of 8.17 $\mathrm{ms}^{-1}$ which was $44.8 \%$ of linear toe velocity at impact. As such, linear velocities at the knee were a good match to the $7 \%$ reported at impact [18] or the maximum of $47 \%$ 
measured at any point in the kick [26] found in the literature. This suggested that the 3$\mathrm{SM}$ was able to match human performance in key areas despite a simplified movement.

One factor affecting the external validity of this research was the simplicity of the anvil simulated. Impact characteristics are dependent on the properties of the impactor and the anvil as both act in-series [9]. However, designing a high-biofidelity anvil is an exhaustive process, one which deserves its own specific attention, and the focus of this research was on improving the impactor. As such, a simplified Lobdell model seemed appropriate as it has been used in the study of thoracic injuries including during a TKD kick [18]. To verify that the values measured here were reasonable, values were compared to data obtained during a pilot study using similar elite martial artists to produce impact forces in vivo on the upper abdominal region of a human subject. This had been done as part of a broader project covered by a generic ethics protocol for the whole larger human impact study. On average, impact forces were $2.8 \pm 0.6 \mathrm{kN}$ with a maximum of $4.2 \mathrm{kN}$. Though these values were slightly lower than those obtained in the $16 \mathrm{~ms}^{-1} 3-\mathrm{SM}$ robot, this would be expected since there was more compliance and energy dissipation, through soft tissue motion and foot compliance, in the human-onhuman impacts. Forces from the STIMAK robot were still substantially lower than those measured by O'Sullivan et al. [27] providing support that the overall system used in this study was more biofidelic than a system consisting of a human kick and sandbag.

Whilst there is support for the biofidelity of the system used here, without an exact replica of the anvil used during human performance it is difficult to compare results directly. As such, a theoretical approach must be taken. First, replacing wobbly soft tissue mass with rigid mass would increase the impact intensity through increased effective mass and decreased energy dissipation [20, 22]. However, the extent of this phenomenon was unclear in a rotational impact, particularly with multiple joints and segments. Second, spring-damper parameters were set to produce correct kinematics pre- impact, but it was unknown how closely they matched the stiffness during impact. This could have a pronounced effect on the contribution of the proximal segments to effective mass as it would help to dissipate part of the impact energy. As such, it was possible that the spring-dampers could offset the exclusion of soft tissue and the 3-SM may be a more effective model than the results were thought to be able to suggest.

The design of the 3-SM model showed that it was not only possible to construct a multi-segment STIMAK robot, but to do so with elements which may increase its biofidelity. The low maximum stress concentrations in the 3-SM FE analysis suggested that it was possible to replace a fraction of the rigid mass with wobbling masses placed strategically (e.g. based on human soft tissue distribution) on each segment. This would help to increase the overall biofidelity of the mechanical impactor and the introduction of wobbling mass effects on the impact response could be determined. At present, this was not possible for the single segment STIMAK as the materials were not strong enough to support a reduction in rigid mass. This design was already optimised to obtain the greatest ratio of strength-to-mass to decrease the inertia of the impactor. As such, there is more scope for improvements in the construction of a multi-segment STIMAK robot in the future. 


\section{Conclusion}

Multi-segment mechanical impactors can be simple yet still replicate complex human movement such as a roundhouse kick in TKD. Using simple segments and joints, data in the flexion-extension axis can be evaluated to obtain the key components of the impact. At three kick velocities (12.0, 14.0, $16.0 \mathrm{~ms}^{-1}$ ), a multi-segment mathematical model was able to match the co-ordination of the knee and ankle at impact producing linear velocities of the knee $(8.4 \%)$ and ankle $(44.8 \%)$ which were close to human performance ( $7 \%$ and $47 \%$, respectively). Moreover, introducing additional segments was able to reduce maximum stress concentrations in the impactor by $\sim 68 \%$ allowing for future development. Properly addressing the co-ordination and masses of an impactor could help to improve upon the future design of PPE to allow evaluations to be conducted more closely to competition conditions. 


\section{References}

1 Nigg, B. M . and Yeadon, M. R. Biomechanical aspects of playing surfaces. J.Sports Sci., 1987, 5, 117-145.

2 Ronkainen, J. A., El-Kati, R. F., Fleming, P. R. and Forrester, S. E. Application of an industrial robot in the sports domain: simulating the ground contact phase of running. In Proc. IMechE, Part P: Journal of Sports Engineering and Technology, 2010, 224 (4), 259-269.

3 Phillpens, $\mathbf{M}$. and Wismans, J. Shin guard impact protection. Proceedings of the IRCOBI Conference, Stockholm, Sweden, 13-15 September 1989, 650-676.

4 Lees, A. and Cooper, S. The shock attenuation characteristics of soccer shinguards. In Sport Leisure and Ergonomics (Eds G. Atkinson and T. Reilly), 1995, pp. 130-135 (E. \& F. B. Spon., London, UK).

5 Francisco, A. C., Nightingale, R. W., Guilak, F., Glisson, R. R. and Garrett Jr., W. E. Comparison of soccer shin guards in preventing tibia fracture. Am. J. Sports Med., 2000, 28(2), 227-233.

6 Ankrah, S. and Mills, N. J. Performance of football shin guards for direct stud impacts. Sports Engineering, 2003, 6, 1-13.

7 Bir, C. A., Cassatta, S. J. and Janda, D. H. An analysis and comparison of soccer shin guards, Clin. J. Sport Med. 1995, 5(2), 95-99.

8 BS EN 13277-3:2000. Protective equipment for martial arts - Part 3: Additional requirements and test methods for trunk protectors, 2000, (British Standards Institution, London).

9 Pain, M.T.G., Tsui, F., and Cove, S. In vivo determination of the effect of shoulder pads on tackling forces in rugby. J. Sports Sci., 2008, 26(8), 855-862.

10 Gerrard, D. F. The use of padding in rugby union. Sports Medicine, 1998, 25, 329-332.

11 Milburn, P. D., Wilson, B. D. and Chalmers, D. J. Evaluation of body protection clothing in rugby union, 2001 (University of Otago, Dunedin, NZ).

12 Walker, P. J. A Doctoral Thesis, Loughborough University. Unpublished.

13 Lee, J. B. A study of kicking techniques of advanced Korea Taekwondo players, 1998 (Korea Institute of Sport Science, Seoul)

14 Holmes, C.E. A Doctoral Thesis, Loughborough University. 2006

15 Dempster, W. T. Space requirements of the seated operator: geometrical, kinematic and mechanical aspects of the body, with special reference to the limbs. 1955, pp 155-159 (Wright Patterson Air Force Base, Dayton, Ohio).

16 Winter, D. A. Biomechanics and motor control of human movement, 1990 (John Wiley \& Sons, Inc., New York).

17 Kong, P. W., Luk, T. C. and Hong, Y. Difference between taekwondo roundhouse kick executed by the front and back leg - a biomechanical study. Proceedings of $23^{\text {rd }}$ International Symposium of Biomechanics in Sports, Hong Kong, 25-30 June 2000, 268-272.

18 Serina, E. R. and Lieu, D. K. Thoracic injury potential of basic competition taekwondo kicks. J. Biomech, 1991, 24(10), 951-960.

19 Ferris, D. P., Louie, M. and Farley, C.T. Running in the real world: adjusting leg stiffness for different surfaces. Proc. R. Soc. London, 1998, 265, 989-994. 
20 Pain, M. T. G. and Challis, J. The influence of soft tissue movement on ground reaction forces, joint torques and joint reaction forces in drop landings. $J$. Biomech., 2006, 39(1), 119-124.

21 Pain, M. T. G. and Challis, J. 2004. Wobbling mass influence on impact ground reaction forces: a simulation model sensitivity analysis. J. App. Biomech., 2004, 20, 309-316.

22 Pain, M. T. G. and Challis, J. Soft tissue motion during impacts: Their potential contribution to energy dissipation. J. Biomech, 2002, 18, 231-242.

23 Schempf, H., Kraueter, C. and Blackwell, M. ROBOLEG: A Robotic Soccer-Ball Kicking Leg. Proceedings of $2^{\text {nd }}$ International Conference on Robotics and Automation, 1995, 1314-1318.

24 Doke, J. and Kuo, A. D. A simple mathematical model of karate front kick. Proceedings of $20^{\text {th }}$ International Symposium on Biomechanics, 2005, Cleveland, Ohio, s648.

25 Lobdell, T.E., Kroell, C. K., Schneider, D. C., Hering, W. E. and Nahum, A. M. Impact response of the human thorax. In: Human Impact Response:

Measurement and Stimulation (Eds W. F. King and H. J. Mertz), 1973, pp 201245. (Plenum Press, New York)

26 Tang, W. T., Chang, J. S. and Nien, Y. H. The kinematics characteristics of preferred and non-preferred roundhouse kick in elite taekwondo athletes. J. Biomech., 2007, 40(s2), s708.

27 O’Sullivan, D., Chung, C., Lee, K., Kim, E., Kang, S., Kim, T. and Shin, I. . Measurement and comparison of Taekwondo and Yongmudo turning kick impact force for two target heights. J. Sports Sci. and Med., 2009, 8, 13-16.

$28 \mathrm{Kim}$, Y. K., Kim, Y. H. and Im, S. J. Inter-joint co-ordination in producing kicking velocity of Taekwondo kicks. J. Sports Sci. and Med., 2011, 10, 31-38. 